\title{
On Exact Learning Monotone DNF from Membership Queries
}

\author{
Hasan Abasi \\ Nader H. Bshouty \\ Department of Computer Science \\ Technion, Haifa, Israel
}

\author{
Hanna Mazzawi \\ IBM Research - Haifa \\ Haifa, Israel.
}

May 6, 2014

\begin{abstract}
In this paper, we study the problem of learning a monotone DNF with at most $s$ terms of size (number of variables in each term) at most $r$ ( $s$ term $r$-MDNF) from membership queries. This problem is equivalent to the problem of learning a general hypergraph using hyperedge-detecting queries, a problem motivated by applications arising in chemical reactions and genome sequencing.

We first present new lower bounds for this problem and then present deterministic and randomized adaptive algorithms with query complexities that are almost optimal. All the algorithms we present in this paper run in time linear in the query complexity and the number of variables $n$. In addition, all of the algorithms we present in this paper are asymptotically tight for fixed $r$ and/or $s$.
\end{abstract}

\section{Introduction}

We consider the problem of learning a monotone DNF with at most $s$ terms, where each monotone term contains at most $r$ variables ( $s$ term $r$-MDNF) from membership queries [1]. This is equivalent to the problem of learning a general hypergraph using hyperedge-detecting queries, a problem that is motivated by applications arising in chemical reaction and genome sequencing.

\subsection{Learning Hypergraph}

A hypergraph is $H=(V, E)$ where $V$ is the set of vertices and $E \subseteq 2^{V}$ is the set of edges. The dimension of the hypergraph $H$ is the cardinality of 
the largest set in $E$. For a set $S \subseteq V$, the edge-detecting queries $Q_{H}(S)$ is answered "Yes" or "No", indicating whether $S$ contains all the vertices of at least one edge of $H$. Our learning problem is equivalent to learning a hidden hypergraph of dimension $r$ using edge-detecting queries.

This problem has many applications in chemical reactions and genome sequencing. In chemical reactions, we are given a set of chemicals, some of which react and some which do not. When multiple chemicals are combined in one test tube, a reaction is detectable if and only if at least one set of the chemicals in the tube reacts. The goal is to identify which sets react using as few experiments as possible. The time needed to compute which experiments to do is a secondary consideration, though it is polynomial for the algorithms we present [5]. See [13, 7, 3, 2, 4, 5] for more details and other applications.

\subsection{Previous Results}

In 5, Angluin and Chen presented an deterministic optimal adaptive learning algorithm for learning $s$-term 2-MDNF. They also gave a lower bound of $\Omega\left((2 s / r)^{r / 2}+r s \log n\right)$ for learning the class of $s$-term $r$-MDNF when $r<s$. In [4], Angluin and Chen gave a randomized algorithm for $s$-term $r$-uniform MDNF (the size of each term is exactly $r$ ) that asks $O\left(2^{4 r} s \cdot \operatorname{poly}(r, \log n)\right)$ membership queries. For $s$-term $r$-MDNF where $r \leq s$, they gave a randomized learning algorithm that asks $O\left(2^{r+r^{2} / 2} s^{1+r / 2} \cdot \operatorname{poly}(\log n)\right)$ membership queries.

Literature has also addressed learning some subclasses of $s$-term 2-MDNF. Those classes have specific applications to genome sequencing. See [13, 7, 3, 2, 4, 5. In this paper we are interested in learning the class of all $s$-term $r$-MDNF formulas for any $r$ and $s$.

\subsection{Our Results}

In this paper, we distinguish between two cases: $s \geq r$ and $s<r$.

For $s<r$, we first prove the lower bound $O\left((r / s)^{s-1}+r s \log n\right)$. We then give three algorithms. Algorithm I is a deterministic algorithm that asks $O\left(r^{s-1}+r s \log n\right)$ membership queries. Algorithm II is a deterministic algorithm that asks $O(s \cdot N((s-1 ; r) ; s r)+r s \log n)$ membership queries where $N((s-1 ; r) ; s r)$ is the size of $(s r,(s-1, r))$-cover free family (see Subsection 2.2 for the definition of cover free) that can be constructed in time linear in its size. An $(s r,(s-1, r))$-cover free family of size $(r / s)^{s-1+o(1)}$ is known to exist. For some $r$ and $s$ (for example $r=o(s \log s \log \log s)$ ), 
such a bound can be achieved in linear time and therefore for those cases, algorithm II is almost optimal. Algorithm III is a randomized algorithm that asks

$$
O\left(\left(\begin{array}{c}
s+r \\
s
\end{array}\right) \sqrt{s r} \log (s r)+r s \log n\right)=O\left(\left(\frac{r}{s}\right)^{s-1+o(1)}+r s \log n\right)
$$

membership queries. This algorithm is almost optimal.

For the case $s \geq r$, Angluin and Chen, [5], gave the lower bound $\Omega\left((2 s / r)^{r / 2}+r s \log n\right)$. We give two algorithms that are almost tight. The first algorithm, Algorithm IV, is a deterministic algorithm that asks $(c r s)^{r / 2+1.5}+r s \log n$ membership queries for some constant $c$. The second algorithm, Algorithm V, is a randomized algorithm that asks $\left(c^{\prime} s\right)^{r / 2+0.75}+$ $r s \log n$ membership queries for some constant $c^{\prime}$.

All the algorithms we present in this paper run in time linear in the query complexity and $n$. Additionally, all the algorithms we describe in this paper are asymptotically tight for fixed $r$ and $s$.

The following table summarizes our results. We have removed the term $r s \log n$ from all the bounds to be able to fit this table in this page. Det. and Rand. stands for deterministic algorithm and randomized algorithm, respectively.

\begin{tabular}{|l|c|l|l|c|}
$r, s$ & $\begin{array}{c}\text { Lower Bound } \\
r s \log n+\end{array}$ & Algorithm & $\begin{array}{l}\text { Rand./ } \\
\text { Det. }\end{array}$ & $\begin{array}{c}\text { Upper Bound } \\
r s \log n+\end{array}$ \\
\hline \hline$r>s$ & \multirow{2}{*}{$\left(\frac{r}{s}\right)^{s-1}$} & Alg. I & Det. & $r^{s-1}$ \\
\cline { 3 - 5 } & & Alg. II & Det. & $s \cdot N((s-1 ; r) ; s r)$ \\
\cline { 3 - 5 } & & Alg. III & Rand. & $(\log r) \sqrt{s} e^{s}\left(\frac{r}{s}+1\right)^{s}$ \\
\hline \multirow{2}{*}{$r \leq s$} & \multirow{2}{*}{$\left(\frac{2 s}{r}\right)^{r / 2}$} & Alg. IV. & Det. & $(3 e)^{r}(r s)^{r / 2+1.5}$ \\
\cline { 3 - 5 } & & Alg. IV. & Rand. & $\sqrt{r}(3 e)^{r}(\log s) s^{r / 2+1}$ \\
\hline
\end{tabular}

\section{Definitions and Notations}

For a vector $w$, we denote by $w_{i}$ the $i$ th entry of $w$. For a positive integer $j$, we denote by $[j]$ the set $\{1,2, \ldots, j\}$.

Let $f\left(x_{1}, x_{2}, \ldots, x_{n}\right)$ be a Boolean function from $\{0,1\}^{n}$ to $\{0,1\}$. For an assignment $a \in\{0,1\}^{n}$ we say that $f$ is $\xi$ in $a$ (or $a$ is $\xi$ in $f$ ) if $f(a)=\xi$. We say that $a$ is zero in $x_{i}$ if $a_{i}=0$. For a set of variables $S$, we say that $a$ is zero in $S$ if for every $x_{i} \in S, a$ is zero in $x_{i}$. Denote $X_{n}=\left\{x_{1}, \ldots, x_{n}\right\}$.

For a Boolean function $f\left(x_{1}, \ldots, x_{n}\right), 1 \leq i_{1}<i_{2}<\cdots<i_{k} \leq n$ and $\sigma_{1}, \ldots, \sigma_{k} \in\{0,1\}$ we denote by

$$
\left.f\right|_{x_{i_{1}}=\sigma_{1}, x_{i_{2}}=\sigma_{2}, \cdots, x_{i_{k}}=\sigma_{k}}
$$


the function $f$ when fixing the variables $x_{i_{j}}$ to $\sigma_{j}$ for all $j \in[k]$. We denote by $\left.a\right|_{x_{i_{1}}=\sigma_{1}, x_{i_{2}}=\sigma_{2}, \cdots, x_{i_{k}}=\sigma_{k}}$ the assignment $a$ where each $a_{i_{j}}$ is replaced by $\sigma_{j}$ for all $j \in[k]$. Note that

$$
\left.f\right|_{x_{i_{1}}=\sigma_{1}, x_{i_{2}}=\sigma_{2}, \cdots, x_{i_{k}}=\sigma_{k}}(a)=f\left(\left.a\right|_{x_{i_{1}}=\sigma_{1}, x_{i_{2}}=\sigma_{2}, \cdots, x_{i_{k}}=\sigma_{k}}\right) .
$$

When $\sigma_{1}=\cdots=\sigma_{k}=\xi$ and $S=\left\{x_{i_{1}}, \ldots, x_{i_{k}}\right\}$, we denote

$$
\left.f\right|_{x_{i_{1}}=\sigma_{1}, x_{i_{2}}=\sigma_{2}, \cdots, x_{i_{k}}=\sigma_{k}}
$$

by $\left.f\right|_{S \leftarrow \xi}$. In the same way, we define $\left.a\right|_{S \leftarrow \xi}$. We denote by $1^{n}=(1,1, \ldots, 1) \in$ $\{0,1\}^{n}$.

For two assignments $a, b \in\{0,1\}^{n}$, we write $a \leq b$ if for every $i, a_{i} \leq b_{i}$. A Boolean function $f:\{0,1\}^{n} \rightarrow\{0,1\}$ is monotone if for every two assignments $a, b \in\{0,1\}^{n}$, if $a \leq b$ then $f(a) \leq f(b)$. Recall that every monotone Boolean function $f$ has a unique representation as a reduced monotone DNF. That is, $f=M_{1} \vee M_{2} \vee \cdots \vee M_{s}$ where each monomial $M_{i}$ is an ANDs of input variables, and for every monomial $M_{i}$ there is a unique assignment $a^{(i)}$ such that $f\left(a^{(i)}\right)=1$ and for every $j \in[n]$ where $a_{j}^{(i)}=1$ we have $f\left(\left.a^{(i)}\right|_{x_{j}=0}\right)=0$. We call such assignment a minterm of the function $f$. Notice that every monotone DNF can be uniquely determined by its minterms.

For a monotone DNF, $f\left(x_{1}, x_{2}, \ldots, x_{n}\right)=M_{1} \vee M_{2} \vee \cdots \vee M_{s}$, and a variable $x_{i}$, we say that $x_{i}$ is $t$-frequent if it appears in more than or equal to $t$ terms. A monotone DNF $f$ is called read $k$ monotone DNF, if none of its variables is $k+1$-frequent.

\subsection{Learning Model}

Consider a teacher (or a black box) that has a target function $f:\{0,1\}^{n} \rightarrow$ $\{0,1\}$ that is $s$-term $r$-MDNF. The teacher can answer membership queries. That is, when receiving $a \in\{0,1\}^{n}$ it returns $f(a)$. A learning algorithm is an algorithm that can ask the teacher membership queries. The goal of the learning algorithm is to exactly learn (exactly find) $f$ with minimum number of membership queries and optimal time complexity.

In our algorithms, for a function $f$ we will denote by $M Q_{f}$ the oracle that answers the membership queries. That is, for $a \in\{0,1\}^{n}, M Q_{f}(a)=f(a)$.

\subsection{Cover-Free Families}

The problem $(n,(s, r))$-cover-free family [12] is equivalent to the following problem: A $(n,(s, r))$-cover-free family is a set $A \subseteq\{0,1\}^{n}$ such that for 
every $1 \leq i_{1}<i_{2}<\cdots<i_{d} \leq n$ where $d=s+r$ and every $J \subseteq[d]$ of size $|J|=s$ there is $a \in A$ such that $a_{i_{k}}=0$ for all $k \in J$ and $a_{i_{j}}=1$ for all $j \notin J$. Denote by $N((s ; r) ; n)$ the minimum size of such set. The lower bounds in [16] are

$$
N((s ; r) ; n) \geq \Omega\left(\frac{(s+r)}{\log \left(\begin{array}{c}
s+r \\
s
\end{array}\right)}\left(\begin{array}{c}
s+r \\
s
\end{array}\right) \log n\right) .
$$

It is known that a set of random

$$
m=O\left(\sqrt{\min (r, s)}\left(\begin{array}{c}
s+r \\
s
\end{array}\right)\left((s+r) \log n+\log \frac{1}{\delta}\right)\right)
$$

vectors $a^{(i)} \in\{0,1\}^{n}$, where each $a_{j}^{(i)}$ is 1 with probability $r /(s+r)$, is a $(n,(s, r))$-cover free family with probability at least $1-\delta$.

In [8], Bshouty gave a deterministic construction of $(n,(s, r))$-CFF of size

$$
\begin{aligned}
C & :=\min \left((2 e)^{s} r^{s+3},(2 e)^{r} s^{r+3}\right) \log n \\
& =\left(\begin{array}{c}
s+r \\
r
\end{array}\right) 2^{\min (s \log s, r \log r)(1+o(1))} \log n
\end{aligned}
$$

that can be constructed in time $C \cdot n$. Fomin et. al. in [11] gave a construction of size

$$
D:=\left(\begin{array}{c}
s+r \\
r
\end{array}\right) 2^{O\left(\frac{r+s}{\log \log (r+s)}\right)} \log n
$$

that can be constructed in time $D \cdot n$. The former bound, (2), is better than the latter when $s \geq r \log r \log \log r$ or $r \geq s \log s \log \log s$. We also note that the former bound, (2), is almost optimal, i.e.,

$$
\left(\begin{array}{c}
s+r \\
r
\end{array}\right)^{1+o(1)} \log n,
$$

when $r=s^{\omega(1)}$ or $r=s^{o(1)}$ and the latter bound, (3) , is almost optimal when

$$
o(s \log \log s \log \log \log s)=r=\omega\left(\frac{s}{\log \log s \log \log \log s}\right) .
$$

\section{Lower Bounds}

In this section, we prove some lower bounds. 


\subsection{General Lower Bound}

In this section, we prove that the information theoretic lower bound for learning a class $C$ from membership queries is also a lower bound for any randomized learning algorithm. We believe it is a folklore result, but we could not find the proof in the literature. We first state the following informationtheoretic lower bound for deterministic learning algorithm,

Lemma 1. Let $C$ be any class of Boolean function. Then any deterministic learning algorithm for $C$ must ask at least $\log |C|$ membership queries.

We now prove,

Lemma 2. Let $C$ be any class of boolean function. Then any Monte Carlo (and therefore, Las Vegas) randomized learning algorithm that learns $C$ with probability at least 3/4 must ask at least $\log |C|-1$ membership queries.

Proof. Let $\mathcal{A}$ be a randomized algorithm that for every $f \in C$ and an oracle $M Q_{f}$ that answers membership queries for $f$, asks $m$ membership queries and satisfies

$$
\operatorname{Pr}_{s}\left[\mathcal{A}\left(M Q_{f}, s\right)=f\right] \geq \frac{3}{4}
$$

where $s \in\{0,1\}^{N}$ is chosen randomly uniformly for some large $N$. Consider the random variable $X_{f}(s)$ that is 1 if $\mathcal{A}\left(M Q_{f}, s\right)=f$ and 0 , otherwise. Then for every $f, \mathbf{E}_{s}\left[X_{f}\right] \geq 3 / 4$. Therefore, for random uniform $f \in C$

$$
3 / 4 \leq \mathbf{E}_{f}\left[\mathbf{E}_{s}\left[X_{f}\right]\right]=\mathbf{E}_{s}\left[\mathbf{E}_{f}\left[X_{f}(s)\right]\right] .
$$

and by Markov Bound for at least $1 / 2$ of the elements $s \in\{0,1\}^{N}$ we have $\mathbf{E}_{f}\left[X_{f}(s)\right] \geq 1 / 2$. Let $S \subseteq\{0,1\}^{N}$ be the set of such elements. Then $|S| \geq$ $2^{N} / 2$. Let $s_{0} \in S$ and $C_{s_{0}} \subseteq C$ the class of functions $f$ where $X_{f}\left(s_{0}\right)=1$. Then $\left|C_{s_{0}}\right| \geq|C| / 2$ and $\mathcal{A}\left(M Q_{f}, s_{0}\right)$ is a deterministic algorithm that learns the class $C_{s_{0}}$. Using the information theoretic lower bound for deterministic algorithm, we conclude that $\mathcal{A}\left(M Q_{f}, s_{0}\right)$ must ask at least

$$
m \geq \log \left|C_{s_{0}}\right|=\log (1 / 2)+\log |C|
$$

membership queries.

Specifically, we have,

Corollary 3. Any Monte Carlo (and therefore Las Vegas) randomized learning algorithm for the class of s-term $r$-MDNF must ask on average at least rs $\log n$ membership queries. 


\subsection{Two Lower Bounds}

In this section, we give two lower bounds. The first is from [4] and the second follows using the same techniques used in [9].

In [4], Angluin and Chen proved,

Theorem 4. Let $r$ and $s$ be integers. Let $k$ and $\ell$ be two integers such that

$$
\ell \leq r, s \geq\left(\begin{array}{l}
k \\
2
\end{array}\right) \ell+1
$$

Any (Monte Carlo) randomized learning algorithm for the class of s-term $r$-MDNF must ask at least

$$
k^{\ell}-1
$$

membership queries.

Specifically, when $s>>r$ we have the lower bound

$$
\Omega\left(\left(\frac{2 s}{r}\right)^{r / 2}\right)
$$

membership queries. Also, for any integer $\lambda$ where

$$
\left(\begin{array}{l}
\lambda \\
2
\end{array}\right) r+1 \leq s<\left(\begin{array}{c}
\lambda+1 \\
2
\end{array}\right) r
$$

we have the lower bound $\lambda^{r}-1$.

We now prove the following lower bound,

Theorem 5. Let $r$ and $s$ be integers and $\ell$ and $t$ be two integers such that

$$
\ell-\left\lfloor\frac{\ell}{t}\right\rfloor \leq r, \quad\left\lfloor\frac{\ell}{t}\right\rfloor \leq s-1 .
$$

Any (Monte Carlo) randomized learning algorithm for the class of s-term $r$-MDNF must ask at least $t^{\lfloor\ell / t\rfloor}$ membership queries.

Specifically, for $r>>s$ we have the lower bound

$$
\left(\frac{r}{s}\right)^{s-1}
$$

and for any constant integer $\lambda$ and $\lambda s \leq r<(\lambda+1) s$ we have the lower bound

$$
(\lambda+1)^{s-1} .
$$


Proof. Let $m=\lfloor\ell / t\rfloor$. Consider the monotone terms $M_{j}=x_{(j-1) t+1} \cdots x_{j t}$ for $j=1,2, \ldots, m$. Define $M_{i, k}$ where $i=1, \ldots, m$ and $k=1, \ldots, t$ the monotone term $M_{i}$ without the variable $x_{(i-1) t+k}$. Let $M_{k_{1}, k_{2}, \ldots, k_{m}}=$ $M_{1, k_{1}} M_{2, k_{2}} \cdots M_{m, k_{m}}$. The only way we can distinguish between the two hypothesis $f=M_{1} \vee M_{2} \vee \cdots \vee M_{m}$ and $g=M_{1} \vee M_{2} \vee \cdots \vee M_{m} \vee M_{k_{1}, k_{2}, \ldots, k_{m}}$ is by guessing an assignment that is 1 in all its first $m t$ entries except for the entire $k_{1}, t+k_{2}, 2 t+k_{3}, \ldots,(m-1) t+k_{m}$. That is, by guessing $k_{1}, k_{2}, \ldots, k_{m}$. This takes an average of $t^{m}$ guesses. Since both $f$ and $g$ are $s$-term $r$-MDNF, the result follows.

For $r>s$, we choose $\ell=r$ and $t$ such that $\lfloor\ell / t\rfloor=s-1$. Since $s-1=\lfloor\ell / t\rfloor \geq \ell / t-1$, we have $t \geq r / s$ and the result follows.

For $\lambda s \leq r<(\lambda+1) s$, proving the lower bound for $r=\lambda s$ is sufficient. Take $t=\lambda+1$ and $\ell=(\lambda+1) s-1$.

\section{Optimal Algorithms for Monotone DNF}

In this section, we present the algorithms (Algorithm I-V) that learn the class of $s$-term $r$-MDNF. We first give a simple algorithm that learns one term. We then give three algorithms (Algorithm I-III) for the case $r>s$ and two algorithms (Algorithm IV-V) for the case $s \geq r$.

\subsection{Learning One Monotone Term}

In this section, we prove the following result.

Lemma 6. Let $f(x)=M_{1} \vee M_{2} \vee \cdots \vee M_{s}$ be the target function where each $M_{i}$ is a monotone term of size at most $r$. Suppose $g(x)=M_{1} \vee M_{2} \vee \cdots \vee M_{s^{\prime}}$ and $h(x)=M_{s^{\prime}+1} \vee M_{s^{\prime}+2} \vee \cdots \vee M_{s}$. If $a$ is an assignment such that $g(a)=0$ and $h(a)=1$, then a monotone term in $h(x)$ can be found with

$$
O\left(r \log \frac{n}{r}\right)
$$

membership queries.

Proof. First notice that since $g$ is monotone, for any $b \leq a$ we have $g(b)=0$. Our algorithm finds a minterm $b \leq a$ of $f$ and therefore $b$ is a minterm of $h$.

First, if the number of ones in $a$ is $2 r$, then we can find a minterm by flipping each bit in $a$ that does change the value of $f$ and get a minterm. This takes at most $2 r$ membership queries.

If the number of ones in $a$ is $w>2 r$, then we divide the entries of $a$ that are equal to 1 into $2 r$ disjoint sets $S_{1}, S_{2}, \ldots, S_{2 r}$ where for every $i$, the size 
of $S_{i}$ is either $\lfloor w /(2 r)\rfloor$ or $\lceil w /(2 r)\rceil$. Now for $i=1,2, \ldots, 2 r$, we flip all the entries of $S_{i}$ in $a$ to zero and ask a membership query. If the function is one, we keep those entries 0 . Otherwise we set them back to 1 and proceed to $i+1$. At the end of this procedure, at most $r$ sets are not flipped. Therefore, at least half of the bits in $a$ are flipped to zero using $2 r$ membership queries. Therefore, the number of membership queries we need to get a minterm is $2 r \log (n / 2 r)+2 r$.

We will call the above procedure Find-Term.

\subsection{The case $r>s$}

In this section, we present three algorithms, two deterministic and one randomized. We start with the deterministic algorithm.

\subsubsection{Deterministic Algorithm}

Consider the class $s$-term $r$-MDNF. Let $f$ be the target function. Given $s-\ell$ monotone terms $M_{1} \vee M_{2} \vee \cdots \vee M_{s-\ell}$ that are known to the learning algorithm to be in $f$. The learning algorithm goal is to find a new monotone term. In order to find a new term we need to find an assignment $a$ that is zero in $M_{1} \vee M_{2} \vee \cdots \vee M_{s-\ell}$ and 1 in the function $f$. Then by the procedure Find-Term in Subsection 4.1, we get a new term in $O(r \log n)$ additional membership queries.

To find such an assignment, we present three algorithms:

Algorithm I: (Exhaustive Search) choose a variable from each $M_{i}$ and set it to zero and set all the other variables to 1 . The set of all such assignments is denoted by $A$. If $f$ is 1 in some $a \in A$, then find a new term using Find-Term.

We now show,

Lemma 7. If $f \not \equiv h$, then Algorithm I finds a new term in $r^{s-\ell}+O(r \log n)$ membership queries.

Proof. Since the number of variables in each term in $h:=M_{1} \vee M_{2} \vee \cdots \vee M_{s-\ell}$ is at most $r$ the number of assignments in $A$ is at most $r^{s-\ell}$. Since we choose one variable from each term in $h$ and set it to zero, all the assignments in $A$ are zero in $h$. We now show that one of the assignments in $A$ must be 1 in $f$, and therefore a new term can be found.

Let $b$ be an assignment that is 1 in $f$ and zero in $h$. Such assignment exists because otherwise $f \Rightarrow h$ and since $h \Rightarrow f$ we get $f \equiv h$. Since 
$h(b)=0$ there is at least one variable $x_{j_{i}}$ in each $M_{i}$ that is zero in $b$. Then the assignment $a:=\left.1^{n}\right|_{x_{j_{1}}=0, \ldots, x_{j_{s-\ell}}=0}$ is in $A$ and $h(a)=0$. Since $a \geq b$ we also have $f(a)=1$.

The number of queries in this algorithm is

$$
\sum_{\ell=1}^{s} O\left(r^{s-\ell}+r \log n\right)=O\left(r^{s-1}+r s \log n\right)
$$

We now present the second algorithm. Recall that $X_{n}=\left\{x_{1}, \ldots, x_{n}\right\}$.

\section{Algorithm II}

1) Let $V$ be the set of variables that appear in $M_{1} \vee M_{2} \vee \cdots \vee M_{s-\ell}$.

2) Take a $(|V|,(s-\ell, r))$-CFF $A$ over the variables $V$.

3) For each $a \in A$

3.1) Define an assignment $a^{\prime}$ that is $a_{i}$ in $x_{i}$ for every $x_{i} \in V$ and 1 in $x_{i}$ for every $x_{i} \in X_{n} \backslash V$.

3.2) If $M_{1} \vee M_{2} \vee \cdots \vee M_{s-\ell}$ is 0 in $a^{\prime}$ and $f$ is one in $a^{\prime}$ then find a new term using Find-Term

Figure 1: Algorithm II for the case $r>s$.

We now show,

Lemma 8. If $f \not \equiv h$, then Algorithm II finds a new term in $N((s-$ $\ell, r),(s-\ell) r)+O(r \log n)$ membership queries.

Proof. Let $h:=M_{1} \vee M_{2} \vee \cdots \vee M_{s-\ell}$. Let $b$ be an assignment that is 1 in $f$ and zero in $h$. Since $h(b)=0$, there is at least one variable $x_{j_{i}}$ in each $M_{i}$ that is zero in $b$. Consider the set $U=\left\{x_{j_{i}} \mid i=1, \ldots, s-\ell\right\}$. Since $f(b)=1$ there is a new term $M$ in $f$ that is one in $b$. That is, all of its variables are one in $b$. Let $W$ be the set of all variables in $M$. Since $A$ is $(|V|,(s-\ell, r))$ $\mathrm{CFF}$ and since $|U \cup(W \cap V)| \leq s-\ell+r$ there is an assignment $a \in A$ that is 0 in each variable in $U$ and is one in each variable in $W \cap V$. Since $a^{\prime}$ is also 0 , in each variable in $U$ we have $h\left(a^{\prime}\right)=0$. Since $a^{\prime}$ is one in each variable in $W \cap V$ and one in each variable $W \backslash V$, we have $M\left(a^{\prime}\right)=1$ and therefore $f\left(a^{\prime}\right)=1$. This completes the proof.

The number of queries in Algorithm II is

$$
\sum_{\ell=1}^{s-1} N((s-\ell, r),(s-\ell) r)+r \log n=O(s N((s-1, r), s r)+r s \log n) \text {. }
$$




\subsubsection{Randomized Algorithm}

Our third algorithm, Algorithm III, is a randomized algorithm. It is basically Algorithm II where an $(r s,(s-1, r))$-CFF $A$ is randomly constructed, as in (11). Notice that an $(r s,(s-1, r))$-CFF is also an $(|V|,(s-\ell, r))-\mathrm{CFF}$, so it can be used in every round of the algorithm. The algorithm fails if there is a new term that has not been found and this happens if and only if $A$ is not $(r s,(s-1, r))$-CFF. So the failure probability is $\delta$. By (1), this gives a Monte Carlo randomized algorithm with query complexity

$$
O\left(\sqrt{s}\left(\begin{array}{c}
s+r \\
s
\end{array}\right)\left(r \log r+\log \frac{1}{\delta}\right)+r s \log n\right) .
$$

\subsection{The case $r<s$}

In this section, we present two algorithms. Algorithm IV is deterministic and Algorithm V is randomized. We start with the deterministic algorithm.

\subsubsection{Deterministic Algorithm}

In this section, we present Algorithm IV, used when $r<s$. For this case, we prove the following,

Theorem 9. There is a deterministic learning algorithm for the class of s-term $r$-MDNF that asks

$$
O\left((3 e)^{r}(r s)^{r / 2+1.5}+r s \log n\right)
$$

membership queries.

Before proving this theorem, we first prove learnability in simpler settings. We prove the following,

Lemma 10. Let $f\left(x_{1}, x_{2}, \ldots, x_{n}\right)=M_{1} \vee \cdots \vee M_{s}$ be the target s-term $r$-MDNF. Suppose the learning algorithm knows some of the terms, $h=$ $M_{1} \vee M_{2} \vee \cdots \vee M_{s-\ell}$ and knows that $M_{s-\ell+1}$ is of size $r^{\prime}$. Suppose that $h$ is a read $k$ monotone DNF. Then, there exists an algorithm that finds a new term (not necessarily $M_{s-\ell+1}$ ) using

$$
\left.O\left(N\left(\left(r^{\prime} k ; r^{\prime}\right) ; s r\right)\right)+r \log n\right),
$$

membership queries. 
LearnRead $\left(M Q_{f}, s, \ell, r^{\prime}\right)$

1) Let $V$ be the set of variables that appear in $h$.

2) Let $A$ be a $\left(|V|,\left(r^{\prime} k, r^{\prime}\right)\right)$-CFF over the variables $V$.

3) For each $a \in A$

3.1) Let $a^{\prime} \in\{0,1\}^{n}$ where $a^{\prime}$ is $a_{i}$ in each $x_{i} \in V$, and one in each $x_{i} \in X_{n} \backslash V$.

3.2) $X \leftarrow \varnothing$.

3.3) For each $M_{i}, i=1, \ldots, s-\ell$ such that $M_{i}\left(a^{\prime}\right)=1$ do

Take any variable $x_{j}$ in $M_{i}$ and set $X \leftarrow X \cup\left\{x_{j}\right\}$

3.4) Set $\left.a^{\prime \prime} \leftarrow a^{\prime}\right|_{X \leftarrow 0}$.

3.5) If $f\left(a^{\prime \prime}\right)=1$ and $h\left(a^{\prime \prime}\right)=0$ then find a new term using Find-Term.

Figure 2: Finding a new term in read $k$.

Proof. Consider the algorithm in Figure 2,

Let $V$ be the set of variables that appear in $h$. Let $M:=M_{s-\ell+1}$. Let $U$ be the set of variables in $M$ and $W=U \cap V$. Each variable in $W$ can appear in at most $k$ terms in $h$. Let w.l.o.g $h^{\prime}:=M_{1} \vee \cdots \vee M_{t}$ be those terms. Notice that $t \leq|W| k \leq r^{\prime} k$. In each term $M_{i}, i \leq t$ one can choose a variable $x_{j_{i}}$ that is not in $W$. This is because, if all the variable in $M_{i}$ are in $W$, then $M \Rightarrow M_{i}$ and then $f$ is not reduced MDNF.

Let $Z=\left\{x_{j_{i}} \mid i=1, \ldots, t\right\}$. Since $|Z| \leq t \leq r^{\prime} k$ and $|U| \leq r^{\prime}$ there is $a \in A$ that is 0 in every variable in $Z$ and is 1 in every variable in $U$. Now notice that $a^{\prime}$ in step 3.1 in the algorithm is the same as $a$ over the variables in $Z$ and therefore $h^{\prime}\left(a^{\prime}\right)=0$. Also $a^{\prime}$ is the same as $a$ over the variables in $U$ and therefore $M\left(a^{\prime}\right)=1$. Now notice that since $M_{i}\left(a^{\prime}\right)=0$ for $i \leq t$, in step 3.4 in the algorithm we only flip $a_{i}^{\prime}$ that correspond to variables in the terms $M_{i}, i>t$. The set of variables in each other term $M_{i}, i>t$ is disjoint with $U$. Therefore if for some $i>t, M_{i}\left(a^{\prime}\right)=1$ then setting any variable $x_{j}$ in $M_{i}$ that is one in $a^{\prime}$ to zero will not change the values $M\left(a^{\prime}\right)=1$ and (from monotonicity) $h^{\prime}\left(a^{\prime}\right)=0$. Eventually, we will have an assignment $a^{\prime \prime}$ that satisfies $h\left(a^{\prime \prime}\right)=0$ and $M\left(a^{\prime \prime}\right)=1$ which implies $f\left(a^{\prime \prime}\right)=1$.

In the following lemma, we remove the restriction on $h$.

Lemma 11. Let $f\left(x_{1}, x_{2}, \ldots, x_{n}\right)=M_{1} \vee \cdots \vee M_{s}$ be the target $s$-term $r$ MDNF. Suppose some of the terms, $h=M_{1} \vee M_{2} \vee \ldots \vee M_{s-\ell}$, are already known to the learning algorithm. Then, for any integer $d$, there exists an 
algorithm that finds a new term using

$$
O\left(\sum_{i=1}^{r}\left(\begin{array}{c}
r \sqrt{d s} \\
i
\end{array}\right) N(((r-i) \sqrt{s / d} ;(r-i)) ; r s)+r \log n\right)
$$

membership queries.

Proof. Consider the algorithm in Figure 3.

$\operatorname{Learn}(s, \ell)$

1) Let $S$ be the set of $\sqrt{s / d}$-frequent variables in $h$.

2) For every $R \subseteq S$ of size $|R| \leq r$ do

2.1) Define $A \in\left(\{0,1\} \cup X_{n}\right)^{n}$ that is 1 in $R$ and 0 in $S \backslash R$ and $A_{i}=x_{i}$ for every $x_{i} \notin S$.

2.2) Run LearnRead $\left(M Q_{f(A)}, s, \ell, r-|R|\right)$ to find $a^{\prime \prime}$.

3) Use $\left.a^{\prime \prime}\right|_{R \leftarrow 1, S \backslash R \leftarrow 0}$ to find a new term using Find-Term.

Figure 3: Finding a new term.

First note that in step 2.2, $f(A)$ is considered in LearnRead as a function in all the variables $X_{n}$. Note also that the oracle $M Q_{f(A)}$ can be simulated by $M Q_{f}$, since $f(A)(a)=f\left(\left.a\right|_{R \leftarrow 0, S \backslash R \leftarrow 1}\right)$.

Let $W$ be the set of variables that appear in $M:=M_{s-\ell+1}$ and $R=$ $S \cap W$. Note that $A$ is zero in all $S \backslash R$ and 1 in $R$ and therefore $f(A)$ is now a $\operatorname{read} \sqrt{s / d}$ and $M(A)$ contains at most $|W \backslash R| \leq r-|R|$ variables. Therefore, when we run LearnRead $\left(M Q_{f(A)}, s, \ell, r-|R|\right)$ we find an assignment $a^{\prime \prime}$ that is 1 in $M(A)$ and zero in $f(A)$ and then $\left.a^{\prime \prime}\right|_{R \leftarrow 0, S \backslash R \leftarrow 1}$ is one in $f$ and zero in $h$.

We now find the number of queries. By the Pigeon hole principle, there are at most $|S| \leq r \sqrt{d s}$ that are $\sqrt{s / d}$-frequent. The number of sets $R \subseteq S$ of size $i$ is $\left(\begin{array}{c}r \sqrt{d s} \\ i\end{array}\right)$. For each set, we run LearnRead $\left(M Q_{f(A)}, s, \ell, r-|R|\right)$ that by Lemma 10 asks $N(((r-i) \sqrt{s / d} ;(r-i)) ; r s)$ queries. This implies the result.

We now prove our main result. We choose $d=r$. Then by the construc- 
tion (2), we have

$$
\begin{aligned}
\left(\begin{array}{c}
r \sqrt{d s} \\
i
\end{array}\right) N(((r-i) \sqrt{s / d} ;(r-i)) ; r s) & \leq\left(\frac{e r \sqrt{r s}}{i}\right)^{i}(2 e)^{r-i}\left(\frac{(r-i) \sqrt{s}}{\sqrt{r}}\right)^{r-i+3} \\
& \leq e^{r} 2^{r-i}(\sqrt{r s})^{r+3}\left(\frac{r}{i}\right)^{i}\left(\frac{r-i}{r}\right)^{r-i+3} \\
& \leq e^{r} 2^{r-i}\left(\begin{array}{c}
r \\
i
\end{array}\right)(\sqrt{r s})^{r+3}
\end{aligned}
$$

and therefore

$$
\sum_{i=1}^{r}\left(\begin{array}{c}
r \sqrt{d s} \\
i
\end{array}\right) N(((r-i) \sqrt{s / d} ;(r-i)) ; r s) \leq(3 e)^{r}(r s)^{r / 2+1.5} .
$$

\subsubsection{Randomized Algorithm}

In this section, we give a randomized algorithm for the case $s>r$.

The randomized algorithm is the same as the deterministic one, except that each CFF is constructed randomly, as in (11) with probability of success $1-\delta / s$. We choose $d=1$ and get

$$
\begin{aligned}
& \left(\begin{array}{c}
r \sqrt{d s} \\
i
\end{array}\right) N(((r-i) \sqrt{s / d} ;(r-i)) ; r s) \\
& \leq\left(\frac{e r \sqrt{s}}{i}\right)^{i} \sqrt{r}(e(\sqrt{s}+1))^{r-i}\left(2 s \log r s+\log \frac{s}{\delta}\right) \\
& \leq e^{r} 2^{r-i}\left(\frac{r}{i}\right)^{i} \sqrt{r} s^{r / 2}(s \log s+\log (1 / \delta))
\end{aligned}
$$

and therefore

$$
\sum_{i=1}^{r}\left(\begin{array}{c}
r \sqrt{d s} \\
i
\end{array}\right) N(((r-i) \sqrt{s / d} ;(r-i)) ; r s) \leq \sqrt{r}(3 e)^{r} s^{r / 2}(s \log s+\log (1 / \delta)) .
$$

\section{Conclusion and Open Problems}

In this paper, we gave an almost optimal adaptive exact learning algorithms for the class of $s$-term $r$-MDNF. When $r$ and $s$ are fixed, the bounds are asymptotically tight. Some gaps occur between the lower bounds and upper bounds. For $r \geq s$, the gap is $c^{s}$ for some constant $c$ and for $r \leq s$ the gap is $r^{r / 2}$. It is interesting to close these gaps. Finding a better deterministic construction of CFF will give better deterministic algorithms. 
Another challenging problem is finding tight bounds for non-adaptive learning of this class.

\section{References}

[1] D. Angluin. Queries and Concept Learning. Machine Learning 2(4), pp. 319-342, (1987).

[2] A. Alon, V. Asodi. Learning a Hidden Subgraph. SIAM J. Discrete Math. 18(4): 697-712 (2005)

[3] N. Alon, R. Beigel, S. Kasif, S. Rudich, B. Sudakov. Learning a Hidden Matching. SIAM J. Comput. 33(2): 487-501 (2004).

[4] D. Angluin, J. Chen. Learning a Hidden Hypergraph. Journal of Machine Learning Research 7, pp. 2215-2236 (2006).

[5] D. Angluin, J. Chen. Learning a hidden graph using $O(\log n)$ queries per edge. J. Comput. Syst. Sci. 74(4): 546-556 (2008)

[6] N. Alon, D. Moshkovitz, S. Safra. Algorithmic construction of sets for $k$ restrictions. ACM Transactions on Algorithms, 2(2), pp. 153-177. (2006).

[7] R. Beigel, N. Alon, S. Kasif, M. Serkan Apaydin, L. Fortnow. An optimal procedure for gap closing in whole genome shotgun sequencing. RECOMB 2001: $22-30$

[8] N. H. Bshouty. Testers and their Applications. Electronic Collouium on Computational Complexity (ECCC) 19:11, (2012). ITCS 2014, pp. 327352.

[9] N. H. Bshouty, S. A. Goldman, Thomas R. Hancock, Sleiman Matar. Asking Questions to Minimize Errors. J. Comput. Syst. Sci. 52(2), pp. 268-286 (1996).

[10] N. H. Bshouty, L. Hellerstein. Attribute-Efficient Learning in Query and Mistakebound Models. COLT (1996), pp. 235-243, (1996).

[11] F. V. Fomin, D. Lokshtanov, S. Saurabh. Efficient Computation of Representative Sets with Applications in Parameterized and Exact Algorithms. SODA 2014, pp. 142-151. (2014).

[12] W. H. Kautz, R. C. Singleton, Nonrandom binary superimposed codes, IEEE Trans. Inform. Theory, 10(4), pp. 363-377, (1964). 
[13] V. Grebinski, G. Kucherov. Reconstructing a Hamiltonian Cycle by Querying the Graph: Application to DNA Physical Mapping. Discrete Applied Mathematics 88(1-3), pp. 147-165 (1998).

[14] D. J. Kleitman, J. Spencer. Families of $k$-independent sets. Discrete Mathematics, 6(3), pp. 255-262, (1972).

[15] M. Naor, L. J. Schulman, A. Srinivasan. Splitters and Near-optimal Derandomization. FOCS 95, pp. 182-191, (1995).

[16] D. R. Stinson, R. Wei, L. Zhu. Some New Bounds for Cover-free Families, Journal of Combinatorial Theory, Series A, 90(1), pp. 224-234, (2000). 